\title{
The Dynamics of an Impulsive Competitive System with Infinite Delay and Diffusion
}

\author{
Hairu Chen, Yuanfu Shao* \\ College of Science, Guilin University of Technology, Guilin, China \\ Email: ^1656233246@qq.com
}

How to cite this paper: Chen, H.R. and Shao, Y.F. (2018) The Dynamics of an Impulsive Competitive System with Infinite Delay and Diffusion. Journal of Applied Mathematics and Physics, 6, 1388-1407. https://doi.org/10.4236/jamp.2018.66116

Received: May 31, 2018

Accepted: June 25, 2018

Published: June 28, 2018

Copyright $\odot 2018$ by authors and Scientific Research Publishing Inc. This work is licensed under the Creative Commons Attribution International License (CC BY 4.0).

http://creativecommons.org/licenses/by/4.0/

\begin{abstract}
In this paper, we consider an impulsive competitive system with infinite delay and diffusion. Firstly, on basis of inequality estimation techniques and comparison theorem of impulsive differential equations, we obtain some sufficient conditions for the permanence and extinction of the system. Then, we establish sufficient conditions for the globally attractive of the system by constructing appropriate Lyapunov function. Besides, under different impulsive conditions, we discuss the effect of time delay and diffusion on dynamic behavior of the competitive system.
\end{abstract}

\section{Keywords}

Diffusion, Infinite Delay, Permanence, Extinction, Attractive

\section{Introduction}

In practical world, owing to many natural and man-made factors (e.g., fire, drought, flooding, crop-dusting, deforestation, hunting, harvesting, etc.), the biological species or ecological environments usually undergo some discrete changes of relatively short duration at some fixed times. Such sudden changes can often be characterized mathematically in the form of impulses. With the development of impulsive differential equations, many experts have adequate mathematical models to investigate the dynamical behaviors of such ecosystems with impulsive effects [1] [2] [3] [4] [5]. On the other hand, the Lotka-Volterra competition systems are very important and significant mathematical models in a non-autonomous environment. Many interesting results of the competitive systems on the existence of positive periodic solutions, permanence, extinction, global stability had been studied extensively (see [6] [7] [8] [9] [10]). For example, Wang [10] investigated the following competitive system with impulsive ef- 
fects:

$$
\left\{\begin{array}{l}
u^{\prime}(t)=u(t)\left(b_{1}(t)-a_{11}(t) u(t)-a_{12}(t) v(t)\right) \\
v^{\prime}(t)=u(t)\left(b_{2}(t)-a_{21}(t) u(t)-a_{22}(t) v(t)\right)
\end{array}\right\}, t \neq t_{k}
$$

The author obtained sufficient conditions on the uniform persistence and extinction of the system by applying the theorem of differential equations.

In ecological environment, because of the natural enemy, severe competition, deterioration of the patch environment, spatial heterogeneity and human activities, species dispersal in two or more patches becomes one of widespread phenomena of nature. It is an important subject to study the effects of dispersion on the dynamics of species living in patchy environments. Many works on population dynamics in patch environment have been investigated [11] [12] [13] [14]. Moreover, in real ecology environment, the existed number on the history will affect indirectly the number of the species at the moment. Therefore, in order to establish more realistic models, the past history of systems should be taken into account, which has led to the introduction of time-delays in differential equations. Such biological system with infinite delay can be found in [15] [16] [17].

Motivated by above arguments, we establish an impulsive competitive system with infinite delay and diffusion as follows:

$$
\left\{\begin{aligned}
x_{1}^{\prime}(t)= & x_{1}(t)\left(a_{1}(t)-b_{1}(t) x_{1}(t)-c_{1}(t) \int_{-\infty}^{0} k_{1}(s) y(t+s) \mathrm{d} s\right) \\
& +D_{12}(t)\left(x_{2}(t)-x_{1}(t)\right) \\
x_{2}^{\prime}(t)= & x_{2}(t)\left(a_{2}(t)-b_{2}(t) x_{2}(t)\right)+D_{21}(t)\left(x_{1}(t)-x_{2}(t)\right) \\
y^{\prime}(t)= & y(t)\left(a_{3}(t)-b_{3}(t) y(t)-c_{2}(t) \int_{-\infty}^{0} k_{2}(s) x_{1}(t+s) \mathrm{d} s\right)
\end{aligned}\right\}, t \neq t_{k}
$$

where $x_{i}(t)(i=1,2)$ and $y(t)$ represent the population densities at time $t$, respectively. Let $0=t_{0}<t_{k}<t_{k+1}, k=1,2, \cdots$ and $t_{k} \rightarrow \infty$ as $k \rightarrow \infty$. Species $x_{1}$ competes with $y$ in patch 1 , while $x_{1}$ can disperse between patch 1 and patch 2 , and $y$ is confined to patch 1. $D_{12}(t), D_{21}(t)$ denotes the diffusion coefficients of species $x . h_{1 k}, h_{2 k}$ and $g_{k}$ are impulsive coefficients at time $t_{k}$, respectively.

We consider system (1.1) with the following initial conditions

$$
\begin{aligned}
& x_{1}(\theta)=\phi_{1}(\theta), \quad x_{2}(\theta)=\phi_{2}(\theta), \quad y(\theta)=\phi_{3}(\theta), \\
& \phi_{i} \in P C_{+}\left(R_{-}, R_{+}\right), i=1,2,3, \quad R_{-}=(-\infty, 0], R_{+}=[0,+\infty) .
\end{aligned}
$$

where $P C_{+}=\left\{\phi=\left(\phi_{1}, \phi_{2}, \phi_{3}\right) \in P C\right\}, \phi_{i}(\theta) \geq 0$ for all $\theta \in R_{-}$and $\phi_{i}\left(0^{+}\right)>0$ 
for $i=1,2,3$. $P C$ is the space of bounded function $\phi(s): R_{-} \rightarrow R^{3}$ which is continuous everywhere expect at the point $t=t_{k} \in I$ and $\phi\left(t_{k}^{+}\right), \phi\left(t_{k}^{-}\right)$exists with $\phi\left(t_{k}^{-}\right)=\phi\left(t_{k}\right)$ and with norm $\|\phi\|=\sup _{\theta \in R_{-}}\left\|\phi_{\theta}\right\|$.

In this paper, for any continuous function $f(t)$, we denote

$$
f(t)=\max _{i \in I}\left\{f_{i}(t)\right\}, \bar{f}(t)=\min _{i \in I}\left\{f_{i}(t)\right\} .
$$

Throughout this paper, we assume that the system (1.1) satisfies ((C1), (C2) see [12], (C3) see [17]):

(C1) all functions are positive, continuous and bounded defined on $R_{+}$, $0<D_{12}(t), D_{21}(t) \leq D$.

(C2) $h_{1 k}, h_{2 k}$ and $g_{k}$ are positive constants for all $k=1,2, \cdots$.

(C3) $k_{i}(s)(i=1,2)$ is a non-negative, piece-wise continuous function defined on $R_{-}$and satisfy $\int_{0}^{+\infty} k_{i}(s) \mathrm{d} s=1$.

Applying some inequality techniques, comparison theorem of impulsive differential equations and Lyapunov function, we study the dynamic behaviors of an impulsive competitive system with infinite delay and diffusion, included permanence, extinction and globally attractive. This paper is organized as follows. Section 2 contains some preliminaries and presents the proof of the lemma. In Section 3, we establish some sufficient conditions which guarantee the system is permanence. In finally section, we give some conditions on the extinction of the system. In Section 4, we study the globally attractive of system (1.1).

\section{Preliminaries}

We consider the following impulsive non-autonomous logistic model

$$
\left\{\begin{array}{l}
x^{\prime}(t)=x(t)(\alpha(t)-\beta(t) x(t)), \quad t \neq t_{k} \\
x\left(t_{k}^{+}\right)=h_{k} x\left(t_{k}\right), \quad k=1,2, \cdots,
\end{array}\right.
$$

where $\alpha(t)$ and $\beta(t)$ are bounded and continuous functions defined on $R_{+}$, $\beta(t) \geq 0$ for all $t \in R_{+}$and impulsive coefficients $h_{k}$ are positive constants for any $k=1,2, \cdots$. Then we have the following Lemma 2.1 .

Lemma 2.1. Suppose that there is a constant $\sigma>0$ such that

$$
\begin{gathered}
\liminf _{t \rightarrow \infty}\left(\int_{t}^{t+\sigma} \beta(s) \mathrm{d} s\right)>0 \\
\liminf _{t \rightarrow \infty}\left(\int_{t}^{t+\sigma} \alpha(s) \mathrm{d} s+\sum_{t \leq t_{k}<t+\sigma} \ln f_{k}\right)>0
\end{gathered}
$$

and function

$$
h(t, \mu)=\sum_{t \leq t_{k}<t+\mu} \ln h_{k}
$$

is bounded on $t \in R_{+}$and $\mu \in[0, \sigma)$. Then we have

1) There exist constant $M>0$ and $m>0$ such that

$$
m \leq \liminf _{t \rightarrow \infty} x(t) \leq \limsup _{t \rightarrow \infty} x(t) \leq M
$$


for any positive solution $x(t)$ of system (2.1).

2) If all conditions of (1) hold, further if

$$
\limsup _{t \rightarrow \infty}\left(\int_{t}^{t+\sigma} \alpha_{1}(s) \mathrm{d} s+\sum_{t \leq t_{k}<t+\sigma} \ln f_{k}\right) \leq 0
$$

then we have $\lim _{t \rightarrow \infty} x(t)=0$ for any positive solution $x(t)$ of system (2.1).

The proof of this Lemma can be found in [18], here we omit it.

Next we consider the following impulsive periodic single species logistic system with diffusion

$$
\left\{\begin{array}{l}
x_{i}^{\prime}(t)=x_{i}(t)\left(r_{i}(t)-a_{i}(t)\right)+\sum_{j=1}^{n} D_{i j}\left(x_{j}(t)-x_{i}(t)\right), t \neq t_{k}, \\
x_{i}\left(t_{k}^{+}\right)=h_{i k} x_{i}\left(t_{k}\right), i=1,2, \cdots, n, k=1,2, \cdots,
\end{array}\right.
$$

Assume that $r_{i}(t), a_{i}(t), D_{i j}(t)(i, j \in I)$ are positive, continuous and bounded functions defined on $R_{+} . D_{2} \geq D_{i j} \geq D_{1}>0(i \neq j), D_{i i}=0$ for all $i, j \in I(I=1,2, \cdots, n)$ and $t \in R_{+}, h_{i k}>0$ for all $i \in I, k=1,2, \cdots$, then we have the following conclusions.

Lemma 2.2. Suppose that there is a positive constant $\bar{\sigma}$ such that

$$
\begin{gathered}
\liminf _{t \rightarrow \infty}\left(\int_{t}^{t+\bar{\sigma}} \bar{a}(s) \mathrm{d} s\right)>0 \\
\liminf _{t \rightarrow \infty}\left(\int_{t}^{t+\bar{\sigma}} \bar{r}(t)-\sum_{j=1}^{n} D_{i j}(t) \mathrm{d} t+\sum_{t \leq t_{k}<t+\bar{\sigma}} \ln \bar{h}_{k}\right)>0
\end{gathered}
$$

and function

$$
h(t, \mu)=\sum_{t \leq t_{k}<t+\mu} \ln h_{k}, \bar{h}(t, \mu)=\sum_{t \leq t_{k}<t+\mu} \ln \bar{h}_{k}
$$

is bounded on $t \in R_{+}$and $\mu \in[0, \bar{\sigma})$. Then we have

1) There are constants $M>0$ and $m>0$ such that

$$
m \leq \liminf _{t \rightarrow \infty} x_{i}(t) \leq \limsup _{t \rightarrow \infty} x_{i}(t) \leq M
$$

for any positive solution $x_{i}(t)$ of $\operatorname{system(2.1).~} t \in R_{+}, i \in I$.

2) If all conditions of (1) hold, further if

$$
\liminf _{t \rightarrow \infty}\left(\int_{t}^{t+\bar{\sigma}} \beta_{1}(t) \mathrm{d} t\right)>0,
$$

where $\beta_{1}(t)=\min _{i \in I}\left\{a_{i}(t)-\sum_{j=1}^{n} \frac{D_{i j}(t)}{m}\right\} \geq 0$ for all $t \in R_{+}, i, j \in I$, then system (2.2) is globally attractive.

Proof: Firstly, we prove system (2.5) is permanent. Let $\left(x_{1}(t), \cdots, x_{i}(t)\right)$ be any solution of system (2.5). Define the function $V_{1}(t)=\max _{i \in I}\left\{x_{i}(t)\right\}$, when $t \neq t_{k}$ calculating the upper-right derivative of $V_{1}(t)$, we have

$$
D^{+} V_{1}(t) \leq x_{i}(t)\left(r_{i}(t)-a_{i}(t) x_{i}(t)\right) \leq V_{1}(t)\left(r(t)-\bar{a}(t) V_{1}(t)\right)
$$

when $t=t_{k}$, we have 


$$
V_{1}\left(t_{k}^{+}\right)=\max _{i \in I}\left\{x_{i}\left(t_{k}^{+}\right)\right\}=\max _{i \in I}\left\{h_{i k} x_{i}\left(t_{k}\right)\right\} \leq \max _{i \in I}\left\{h_{i k}\right\} \max _{i \in I}\left\{x_{i}\left(t_{k}\right)\right\}=h_{k} V_{1}\left(t_{k}\right)
$$

Consider the following auxiliary system

$$
\left\{\begin{array}{l}
D^{+} w(t)=w(t)(r(t)-\bar{a}(t) w(t)), t \neq t_{k} \\
w\left(t_{k}^{+}\right)=h_{k} w\left(t_{k}\right), k=1,2, \cdots
\end{array}\right.
$$

with initial condition $w(0)=V_{1}(0)$. Obviously, from condition (2.6), (2.8) and Lemma 2.1, there exists a constant $M>0$ such that $\limsup w(t) \leq M$. Then according to comparison theorem of impulsive differential equations, we derive

$$
\limsup _{t \rightarrow \infty} x_{i}(t) \leq \limsup _{t \rightarrow \infty} V_{1}(t) \leq \limsup _{t \rightarrow \infty} w(t) \leq M \text { for } i \in I \text {. }
$$

Now we prove there is a constant $m>0$ such that $\liminf _{t \rightarrow \infty} x_{i}(t) \geq m$. Defined $V_{2}(t)=\min _{i \in I}\left\{x_{i}(t)\right\}$, calculating the right-lower derivative of $V_{2}(t)$ when $t \neq t_{k}$, similar to above conclusion, we can obtain

$$
D^{+} V_{2}(t) \geq x_{i}(t)\left(r_{i}(t)-a_{i}(t) x_{i}(t)\right) \geq V_{2}(t)\left(\bar{r}(t)-a(t) V_{2}(t)\right)
$$

when $t=t_{k}$, we have

$$
V_{2}\left(t_{k}^{+}\right)=\min _{i=\{1,2\}}\left\{x_{i}\left(t_{k}^{+}\right)\right\}=\min _{i=\{1,2\}}\left\{h_{i k} x_{i}\left(t_{k}\right)\right\} \geq \min _{i=\{1,2\}}\left\{h_{i k}\right\} \min _{i=\{1,2\}}\left\{x_{i}\left(t_{k}\right)\right\}=\bar{h}_{k} V_{2}\left(t_{k}\right)
$$

by comparison theorem of impulsive differential equations, we derive $V_{2}(t) \geq v(t)$ for all $t \in R_{+}$, where $v(t)$ is the solution of the auxiliary equation

$$
\left\{\begin{array}{l}
D^{+} v(t)=v(t)\left(\bar{r}(t)-a(t) v_{2}(t)\right), t \neq t_{k} \\
v\left(t_{k}^{+}\right)=\bar{h}_{k} v\left(t_{k}\right), k=1,2, \cdots
\end{array}\right.
$$

with initial condition $V_{2}(0)=v(0)$. Clearly, from condition (2.7), (2.8) and Lemma 2.1, there is a constant $m>0$ such that $\liminf _{t \rightarrow \infty} v(t) \geq m$. Therefore we have

$$
\liminf _{t \rightarrow \infty} x_{i}(t) \geq \liminf _{t \rightarrow \infty} V_{2}(t) \geq \liminf _{t \rightarrow \infty} v(t) \geq m \text { for } i \in I .
$$

Next we consider the globally attractive of system (2.5). Construct a Lyapunov function

$$
V(t)=\sum_{i=1}^{n}\left|\ln x_{i}(t)-\ln \tilde{x}_{i}(t)\right|
$$

when $t=t_{k}$, we always have

$$
V\left(t_{k}\right)=\sum_{i=1}^{n}\left|\ln h_{i k} x_{i}\left(t_{k}\right)-\ln h_{i k} \tilde{x}_{i}\left(t_{k}\right)\right|=V\left(t_{k}^{+}\right)
$$

then $V(t)$ is continuous for all $t \in R_{+}$. In addition, when $t \in R_{+}$and $t \neq t_{k}$ we have

$$
\frac{1}{B}\left|x_{i}(t)-\tilde{x}_{i}(t)\right| \leq\left|\ln x_{i}(t)-\ln \tilde{x}_{i}(t)\right| \leq \frac{1}{A}\left|x_{i}(t)-\tilde{x}_{i}(t)\right|
$$

Moreover, calculating the derivative of $V(t)$, we also have

$$
\begin{aligned}
D^{+} V(t) & =\sum_{i=1}^{n} \operatorname{sgn}\left(x_{1}(t)-\tilde{x}_{1}(t)\right)\left(\frac{x_{i}^{\prime}(t)}{x_{i}(t)}-\frac{\tilde{x}_{i}^{\prime}(t)}{\tilde{x}_{i}(t)}\right) \\
& \leq \sum_{i=1}^{n}\left(-a_{i}(t)\left|x_{i}(t)-\tilde{x}_{i}(t)\right|\right)+\sum_{i=1}^{n} \sum_{j=1}^{n} \bar{D}_{i j}(t)
\end{aligned}
$$


where $\bar{D}_{i j}(t)=\left\{\begin{array}{l}D_{i j}(t)\left(\frac{x_{j}(t)}{x_{i}(t)}-\frac{\tilde{x}_{j}(t)}{\tilde{x}_{i}(t)}\right), x_{i}(t)>\tilde{x}_{i}(t), \\ D_{i j}(t)\left(\frac{\tilde{x}_{j}(t)}{\tilde{x}_{i}(t)}-\frac{x_{j}(t)}{x_{i}(t)}\right), x_{i}(t)<\tilde{x}_{i}(t) .\end{array}\right.$

For all $t \in R_{+}$, we think about under the following two cases:

Case 1: if $x_{i}(t)>\tilde{x}_{i}(t)$, then

$$
\bar{D}_{i j}(t) \leq \frac{D_{i j}(t)}{x_{i}(t)}\left(x_{j}(t)-\tilde{x}_{j}(t)\right) \leq \frac{D_{i j}(t)}{A}\left|x_{j}(t)-\tilde{x}_{j}(t)\right|
$$

Case 2: if $x_{i}(t)<\tilde{x}_{i}(t)$, then

$$
\bar{D}_{i j}(t) \leq \frac{D_{i j}(t)}{\tilde{x}_{i}(t)}\left(\tilde{x}_{j}(t)-x_{j}(t)\right) \leq \frac{D_{i j}(t)}{A}\left|x_{j}(t)-\tilde{x}_{j}(t)\right|
$$

From Case 1, Case 2 and (2.14), we can obtain

$$
\begin{aligned}
D^{+} V(t) & \leq \sum_{i=1}^{n}\left(-a_{i}(t)\left|x_{i}(t)-\tilde{x}_{i}(t)\right|\right)+\sum_{i=1}^{n} \sum_{j=1}^{n} \frac{D_{i j}}{A}\left|x_{j}(t)-\tilde{x}_{j}(t)\right| \\
& \leq-\sum_{i=1}^{n}\left(a_{i}(t)-\sum_{j=1}^{n} \frac{D_{i j}}{A}\right)\left|x_{i}(t)-\tilde{x}_{i}(t)\right| \\
& \leq-\beta(t) A V(t)
\end{aligned}
$$

By (2.15) and condition (2.9), we have $V(t) \leq V(0) \exp \left(-A \int_{0}^{t} \beta(s) \mathrm{d} s\right) \rightarrow 0$ as $t \rightarrow \infty$. Furthermore, by (2.13), we have $\lim _{t \rightarrow \infty}\left(x_{i}(t)-\tilde{x}_{i}(t)\right)=0$, that is, system (2.5) has globally attractive positive solution. This completes the proof of Lemma 2.2.

\section{Permanence}

Note that system (1.1) always has a positive solution for all $t \in R_{+}$if it has a positive initial condition. Here we state and prove the permanent of system (1.1).

Theorem 3.1. There exists a constant $M>0$ such that $\limsup x_{i}(t) \leq M$ and $\lim \sup y(t) \leq M$ for any positive solution of system (1.1) if there exists a constant $\stackrel{t \rightarrow \infty}{\infty} \omega>0$ such that

$$
\begin{aligned}
& \liminf _{t \rightarrow \infty}\left(\int_{t}^{t+\omega} \bar{b}(s) \mathrm{d} s\right)>0 \\
& \liminf _{t \rightarrow \infty}\left(\int_{t}^{t+\omega} b_{3}(s) \mathrm{d} s\right)>0
\end{aligned}
$$

and function

$$
h(t, \mu)=\sum_{t \leq t_{k}<t+\nu} \ln h_{k}, g(t, \mu)=\sum_{t \leq t_{k}<t+\mu} \ln g_{k}
$$

are bounded on $t \in R_{+}$and $\mu \in[0, \omega)$ for all $t \in R_{+}, k=1,2, \cdots$.

Proof. Firstly, we prove that there is a constant $M_{1}>0$ such that $\limsup _{t \rightarrow \infty} x_{i}(t) \leq M_{1}(i=1,2)$. Define the function $V(t)=\max \left\{x_{1}(t), x_{2}(t)\right\}$, we 
have two cases when $t \neq t_{k}$.

1) If $V(t)=x_{1}(t)$, we have

$$
\begin{aligned}
D^{+} V(t)= & x_{1}(t)\left(a_{1}(t)-b_{1}(t) x_{1}(t)-c_{1}(t) \int_{-\infty}^{0} k_{1}(s) y(t+s) \mathrm{d} s\right) \\
& +D_{12}(t)\left(x_{2}(t)-x_{1}(t)\right) \\
\leq & x_{1}(t)\left(a_{1}(t)-b_{1}(t) x_{1}(t)\right) \\
\leq & V(t)(a(t)-\bar{b}(t) V(t))
\end{aligned}
$$

2) If $V(t)=x_{2}(t)$, we have

$$
\begin{aligned}
D^{+} V(t) & =x_{2}(t)\left(a_{2}(t)-b_{2}(t) x_{2}(t)\right)+D_{21}(t)\left(x_{1}(t)-x_{2}(t)\right) \\
& \leq x_{2}(t)\left(a_{2}(t)-b_{2}(t) x_{2}(t)\right) \\
& \leq V(t)(a(t)-\bar{b}(t) V(t))
\end{aligned}
$$

clearly, from (3.4) and (3.5), we get

$$
D^{+} V(t) \leq V(t)(a(t)-\bar{b}(t) V(t)) \text { for all } t \in R_{+}
$$

On the other hand, when $t=t_{k}$, we have

$$
V\left(t_{k}^{+}\right)=\max _{i=\{1,2\}}\left\{x_{i}\left(t_{k}^{+}\right)\right\}=\max _{i=\{1,2\}}\left\{h_{i k} x_{i}\left(t_{k}\right)\right\} \leq \max _{i=\{1,2\}}\left\{h_{i k}\right\} \max _{i=\{1,2\}}\left\{x_{i}\left(t_{k}\right)\right\}=h_{k} V\left(t_{k}\right) .
$$

Consider the following auxiliary equation

$$
\left\{\begin{array}{l}
D^{+} v(t)=v(t)(a(t)-\bar{b}(t) v(t)), t \neq t_{k} \\
v\left(t_{k}^{+}\right)=h_{k} v\left(t_{k}\right), k=1,2, \cdots
\end{array}\right.
$$

with initial condition $v(0)=V(0)$. Since the condition of Lemma 2.1 holds from (3.1) and (3.3), using Lemma 2.1, we get that there exists a constant $M_{1}>0$ such that $\limsup v(t) \leq M_{1}$. Applying the comparison theorem of impulsive differential equation, we obtain $V(t) \leq v(t)$ for all $t \in R_{+}$. Finally, we have

$$
\limsup _{t \rightarrow \infty} x_{i}(t) \leq \limsup _{t \rightarrow \infty} V(t) \leq \limsup _{t \rightarrow \infty} v(t) \leq M_{1} \text { for } i=1,2
$$

Then we prove that there is a constant $M_{2}>0$ such that $\limsup y(t) \leq M_{2}$. From the third and sixth equations of system (1.1), we obtain

$$
\left\{\begin{aligned}
y^{\prime}(t) & =y(t)\left(a_{3}(t)-b_{3}(t) y(t)-c_{2}(t) \int_{-\infty}^{0} k_{2}(s) x_{1}(t-s) \mathrm{d} s\right) \\
& \leq y(t)\left(a_{3}(t)-b_{3}(t) y(t)\right), t \neq t_{k} \\
y\left(t_{k}^{+}\right) & =g_{k} y\left(t_{k}\right), k=1,2, \cdots
\end{aligned}\right.
$$

considering the following subsystem

$$
\left\{\begin{array}{l}
u^{\prime}(t)=u(t)\left(a_{3}(t)-b_{3}(t) u(t)\right), t \neq t_{k} \\
u\left(t_{k}^{+}\right)=g_{k} u(t), k=1,2, \cdots
\end{array}\right.
$$

with initial condition $u(0)=y(0)$. Obviously, the condition of Lemma 2.1 holds from (3.2) and (3.3), we obtain that there is a constant $M_{2}>0$ such that 
$\limsup u(t) \leq M_{2}$. Similar to the prove process of the bounded of species $x$, we have $\stackrel{t \rightarrow \infty}{\lim \sup } y(t) \leq M_{2}$.

Let $\stackrel{t \rightarrow \infty}{M}=\max \left\{M_{1}, M_{2}\right\}$, evidently, we have

$$
\limsup _{t \rightarrow \infty} x_{i}(t) \leq M(i=1,2), \quad \limsup _{t \rightarrow \infty} y(t) \leq M
$$

The proof of Theorem 3.1 is completed.

Theorem 3.2. Assume that all conditions of Theorem 3.1 are satisfied. In addition, there is a constant $\bar{\omega}>0$ such that

$$
\begin{gathered}
\liminf _{t \rightarrow \infty}\left(\int_{t}^{t+\bar{\omega}} \bar{a}(s) \mathrm{d} s+\sum_{t \leq t_{k}<t+\bar{\omega}} \ln \bar{h}_{k}\right)>0 \\
\liminf _{t \rightarrow \infty}\left(\int_{t}^{t+\bar{\omega}}\left(a_{3}(t)-c_{2}(t) \int_{-\tau}^{0} k_{2}(s)\left(x_{1}^{*}(t+s)\right) \mathrm{d} s\right) \mathrm{d} s+\sum_{t \leq t_{k}<t+\bar{\omega}} \ln g_{k}\right)>0 \\
\liminf _{t \rightarrow \infty}\left(\int_{t}^{t+\bar{\omega}} \beta_{2}(t) \mathrm{d} t\right)>0
\end{gathered}
$$

and function

$$
\bar{h}(t, \mu)=\sum_{t \leq t_{k}<t+\mu} \ln \bar{h}_{k}, \quad g(t, \mu)=\sum_{t \leq t_{k}<t+\mu} \ln g_{k}
$$

are bounded on $t \in R_{+}$and $\mu \in[0, \bar{\omega})$, where

$\beta_{2}(t)=\min _{i=1,2}\left\{a_{i}(t)-\sum_{j=1}^{2} \frac{D_{i j}(t)}{p}\right\} \geq 0(i \neq j)$ for all $t \in R_{+}$.

Then the system is permanent.

Proof. Above all, we must prove that there exists a constant $m>0$ such that $\liminf _{t \rightarrow \infty} x_{i}(t) \geq m(i=1,2), \quad \liminf _{t \rightarrow \infty} y(t) \geq m$.

Firstly, we prove $\liminf _{t \rightarrow \infty} x_{i}(t) \geq m(i=1,2)$. Defined $V(t)=\min \left\{x_{1}(t), x_{2}(t)\right\}$ and

$$
H=\sup \left\{\left|x_{i}(t+s), y(t+s)\right|: t \in R_{+}, s \in R_{-}, i=1,2\right\}
$$

When $t \neq t_{k}$, consider the following two cases.

Case 1: If $V(t)=x_{1}(t)$, we can choose a constant $\tau>0$ such that $H \int_{-\infty}^{-\tau} k_{1}(s) \mathrm{d} s<M$, then we have

$$
\begin{aligned}
& V^{\prime}(t)= x_{1}(t)\left(a_{1}(t)-b_{1}(t) x_{1}(t)-c_{1}(t) \int_{-\infty}^{0} k_{1}(s) y(t+s) \mathrm{d} s\right) \\
&+D_{12}(t)\left(x_{2}(t)-x_{1}(t)\right) \\
&= x_{1}(t)\left(a_{1}(t)-b_{1}(t) x_{1}(t)-c_{1}(t)\left(\int_{-\infty}^{-\tau} k_{1}(s) y(t+s) \mathrm{d} s\right.\right. \\
&\left.\left.+\int_{-\tau}^{0} k_{1}(s) y(t+s) \mathrm{d} s\right)\right)+D_{12}(t)\left(x_{2}(t)-x_{1}(t)\right) \\
& \geq x_{1}(t)\left(a_{1}(t)-b_{1}(t) x_{1}(t)-c_{1}(t)\left(H \int_{-\infty}^{-\tau} k_{1}(s) \mathrm{d} s+\int_{-\tau}^{0} k_{1}(s) y(t+s) \mathrm{d} s\right)\right) \\
& \geq x_{1}(t)\left(a_{1}(t)-2 c_{1}(t) M-b_{1}(t) x_{1}(t)\right) \\
& \geq V(t)\left(\bar{a}(t)-2 c_{1}(t) M-b(t) V(t)\right),
\end{aligned}
$$

Case 2: If $V(t)=x_{2}(t)$, we have 


$$
\begin{aligned}
V^{\prime}(t) & =x_{2}(t)\left(a_{2}(t)-b_{2}(t) x_{2}(t)\right)+D_{21}(t)\left(x_{1}(t)-x_{2}(t)\right) \\
& \geq x_{2}(t)\left(a_{2}(t)-b_{2}(t) x_{2}(t)\right) \\
& \geq V(t)(\bar{a}(t)-b(t) V(t)) .
\end{aligned}
$$

By Case 1 and Case 2, we derive $V(t) \geq V(t)(\bar{a}(t)-b(t) V(t))$.

When $t=t_{k}$, we can obtain

$$
V\left(t_{k}^{+}\right)=\min _{i=\{1,2\}}\left\{x_{i}\left(t_{k}^{+}\right)\right\}=\min _{i=\{1,2\}}\left\{h_{i k} x_{i}\left(t_{k}\right)\right\} \geq \min _{i=\{1,2\}}\left\{h_{i k}\right\} \min _{i=\{1,2\}}\left\{x_{i}\left(t_{k}\right)\right\}=\bar{h}_{k} V\left(t_{k}\right) .
$$

Research the following equation with impulsive

$$
\left\{\begin{array}{l}
v^{\prime}(t)=v(t)(\bar{a}(t)-b(t) v(t)), t \neq t_{k} \\
v\left(t_{k}^{+}\right)=\bar{h}_{k} v(t), k=1,2, \cdots
\end{array}\right.
$$

By condition (3.9) and (3.12), we know that condition of Lemma 2.1 is satisfied. Consequently, there exists a constant $m_{1}>0$ such that

$$
\liminf _{t \rightarrow \infty} x_{i}(t) \geq \liminf _{t \rightarrow \infty} V(t) \geq \liminf _{t \rightarrow \infty} v(t) \geq m_{1} \text { for } i=1,2 .
$$

Then we investigate the following system:

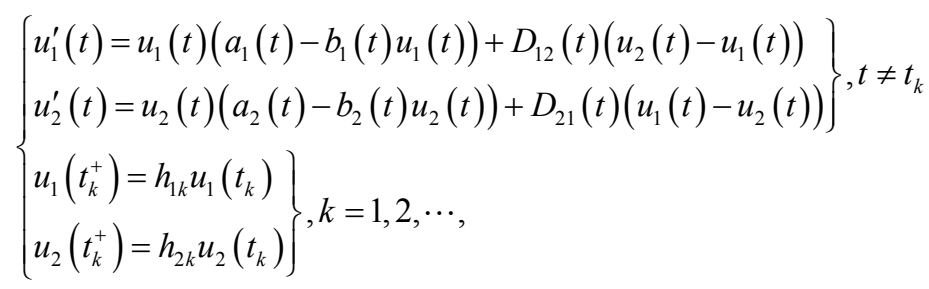

From Lemma 2.2 and condition (3.1), (3.10), (3.12) and (3.13), we can know that there are positive constants $p$ and $P$ such that

$$
p \leq x_{i}^{*}(t) \leq P
$$

where $x_{i}^{*}(t)(i=1,2)$ is globally attractive for the system (3.16). In addition, we assume that $\left(u_{1}(t), u_{2}(t)\right)$ is a positive solution of system (3.16) with initial condition $u_{i}(0)=x_{i}(0)$. Evidently, we obtain that there exists a constant $\varepsilon_{0}>0$ small enough such that

$$
x_{i}^{*}(t)-\varepsilon_{0} \leq u_{i}(t) \leq x_{i}^{*}(t)+\varepsilon_{0}
$$

Similar to the discussion in [18], we obtain that condition (3.10) is independent of the choice of $x_{i}^{*}(t)$.

From condition (3.10), there are constant $\varepsilon_{0}>0$ small enough and $T>0$ large enough such that

$$
\begin{aligned}
& \int_{t}^{t+\bar{\omega}}\left(a_{3}(t)-b_{3}(t) \varepsilon_{0}-2 c_{2}(t) \varepsilon_{0}-c_{2}(t) \int_{-\tau}^{0} k_{2}(s)\left(x_{1}^{*}(t+s)\right) \mathrm{d} s\right) \mathrm{d} s \\
& +\sum_{t \leq t_{k}<t+\bar{\omega}} \ln g_{k}>\varepsilon_{0}
\end{aligned}
$$

for all $t \geq T$. By (3.12), we can get a positive constant $G$ such that

$$
|g(t, \mu)|=\left|\sum_{t \leq t_{k}<t+\mu} \ln g_{k}\right| \leq G .
$$


From system (1.1), we consider the following subsystem,

$$
\left\{\begin{aligned}
x_{1}^{\prime}(t)= & x_{1}(t)\left(a_{1}(t)-b_{1}(t) x_{1}(t)-c_{1}(t) \int_{-\infty}^{0} k_{1}(s) y(t+s) \mathrm{d} s\right) \\
& +D_{12}(t)\left(x_{2}(t)-x_{1}(t)\right) \\
x_{2}^{\prime}(t)= & x_{2}(t)\left(a_{2}(t)-b_{2}(t) x_{2}(t)\right)+D_{21}(t)\left(x_{1}(t)-x_{2}(t)\right)
\end{aligned}\right\}, t \neq t_{k}
$$

By (3.16), (3.17) and comparison theorem of impulsive differential equations, we get that

$$
x_{1}(t) \leq u_{1}(t) \leq x_{1}^{*}(t)+\varepsilon_{0} \text { for all } t \geq T_{1} \geq T .
$$

Next we prove there is a constant $m_{2}>0$ such that $\liminf _{t \rightarrow \infty} y(t) \geq m_{2}$.

In the beginning, we prove $\lim \sup y(t) \geq \varepsilon_{0}$. Suppose that the proposition is not true, we have $\limsup y(t)<\varepsilon_{0}$, that is $y(t)<\varepsilon_{0}$ for all $t \geq T_{2}>T_{1}$. Furthermore, we can choose a constant $\tau>0$ such that

$$
H \int_{-\infty}^{-\tau} k_{2}(s) \mathrm{d} s \leq \varepsilon_{0}
$$

Consequently, we have

$y^{\prime}(t)$

$\geq y(t)\left(a_{3}(t)-b_{3}(t) y(t)-c_{2}(t)\left(H \int_{-\infty}^{-\tau} k_{2}(s) \mathrm{d} s+\int_{-\tau}^{0} k_{2}(s)\left(x_{1}^{*}(t+s)+\varepsilon_{0}\right) \mathrm{d} s\right)\right)(3.2$

$\geq y(t)\left(a_{3}(t)-b_{3}(t) \varepsilon_{0}-2 c_{2}(t) \varepsilon_{0}-c_{2}(t) \int_{-\tau}^{0} k_{2}(s)\left(x_{1}^{*}(t+s)\right) \mathrm{d} s\right)$

For any $t \geq T_{2}+\tau$ and $t \neq t_{k}$, we can choose an integer $l \geq 0$ such that $t=T_{2}+\tau+l \bar{\omega}+v$, where $v \in[0, \bar{\omega})$ is a constant. Integrating (3.22) from $T_{2}+\tau$ to $t$, due to (3.18) and (3.19), we derive

$$
\begin{gathered}
y(t) \geq y\left(T_{2}+\tau\right) \exp \left(\int _ { T _ { 2 } + \tau } ^ { t } \left(a_{3}(t)-b_{3}(t) y(t)\right.\right. \\
\left.\left.-c_{2}(t) \int_{-\infty}^{0} k_{2}(s) x_{1}(t+s) \mathrm{d} s\right) \mathrm{~d} s+\sum_{T_{2}+\tau \leq t_{k}<t} \ln g_{k}\right) \\
\geq y\left(T_{2}+\tau\right) \exp \left(\int _ { T _ { 2 } + \tau } ^ { t } \left(a_{3}(t)-b_{3}(t) \varepsilon_{0}-2 c_{2}(t) \varepsilon_{0}\right.\right. \\
\left.\left.-c_{2}(t) \int_{-\tau}^{0} k_{2}(s) x_{1}^{*}(t+s) \mathrm{d} s\right)+\sum_{T_{2}+\tau \leq t_{k}<t} \ln g_{k}\right) \\
\geq y\left(T_{2}+\tau\right) \exp \left(\int_{T_{2}+\tau}^{T_{2}+\tau+\bar{\omega}}+\cdots+\int_{T_{2}+\tau+(l-1) \omega}^{T_{2}+\tau+l \bar{\omega}}+\int_{T_{2}+\tau+l \omega}^{t}\left(a_{3}(t)-b_{3}(t) \varepsilon_{0}\right.\right. \\
\left.-2 c_{2}(t) \varepsilon_{0}-c_{2}(t) \int_{-\tau}^{0} k_{2}(s) x_{1}^{*}(t+s) \mathrm{d} s\right) \\
\left.+\sum_{T_{2}+\tau \leq t_{k}<T_{2}+\tau+\bar{\omega}}+\cdots+\sum_{T_{2}+\tau+(l-1) \overline{\bar{\omega}} \leq_{k}<T_{2}+\tau+l \bar{\omega}}+\sum_{T_{2}+\tau+l \bar{\omega} \leq t_{k}<t} \ln g_{k}\right) \\
\geq y\left(T_{2}+\tau\right) \exp \left(l \varepsilon_{0}-\beta \bar{\omega}-G\right),
\end{gathered}
$$


where $\beta=\sup _{t \in R_{+}}\left(\left|a_{3}(t)\right|+b_{3}(t) \varepsilon_{0}+2 c_{2}(t) \varepsilon_{0}+c_{2}(t) \int_{-\tau}^{0} k_{2}(s) x_{1}^{*}(t+s) \mathrm{d} s\right)$. Therefore, we can get $y(t) \rightarrow \infty$ as $t \rightarrow \infty$, which is contradiction with $\limsup y(t)<\varepsilon_{0}$. Obviously, we have

$$
\limsup _{t \rightarrow \infty} y(t) \geq \varepsilon_{0}
$$

Then we prove there exists a constant $\alpha>0$ such that $\liminf _{t \rightarrow \infty} y(t) \geq \alpha$. Assume that the proposition is not true, then there exists a sequence $\left\{\phi_{m}(t)\right\} \in P C_{+}, m=1,2, \cdots$ such that $\liminf _{t \rightarrow \infty} y\left(t, \phi_{k}\right) \leq \frac{\varepsilon_{0}}{k^{2}}$ for all $k=1,2, \cdots$.

From (3.19), we have

$$
\mathrm{e}^{-G} \leq g_{k} \leq \mathrm{e}^{G}
$$

we choose an integer $K>\mathrm{e}^{G}$ such that for all $k \geq K$ and any solution $y(t)$ of system (1.1) satisfied:

1) If $y\left(t_{l}\right) \geq \frac{\varepsilon_{0}}{k}$, then $y\left(t_{l}^{+}\right)=g_{k} y\left(t_{l}\right) \geq \mathrm{e}^{-G} \frac{\varepsilon_{0}}{k} \geq \frac{\varepsilon_{0}}{k^{2}}$, for some $l=1,2, \cdots$,

2) If $y\left(t_{l}\right) \leq \frac{\varepsilon_{0}}{k^{2}}$, then $y\left(t_{l}^{+}\right)=g_{k} y\left(t_{l}\right) \leq \mathrm{e}^{G} \frac{\varepsilon_{0}}{k^{2}} \geq \frac{\varepsilon_{0}}{k}$, for some $l=1,2, \cdots$.

From above inequality, there exist two time sequences $\left\{s_{q}^{(k)}\right\}$ and $\left\{t_{q}^{(k)}\right\}$ such that for each $k=K+1, K+2, \cdots$, we have

$$
0<s_{1}^{(k)}<t_{1}^{(k)}<s_{2}^{(k)}<t_{2}^{(k)}<\cdots<s_{q}^{(k)}<t_{q}^{(k)}<\cdots
$$

and

$$
\begin{gathered}
\left\{s_{q}^{(k)}\right\} \rightarrow \infty,\left\{t_{q}^{(k)}\right\} \rightarrow \infty \text { as } t \rightarrow \infty \\
y\left(s_{q}^{(k)}, \phi_{k}\right) \geq \frac{\varepsilon_{0}}{k}, \frac{\varepsilon_{0}}{k^{2}}<y\left(s_{q}^{(k)+}, \phi_{k}\right) \leq \frac{\varepsilon_{0}}{k}, \\
\frac{\varepsilon_{0}}{k^{2}} \leq y\left(t_{q}^{(k)}, \phi_{k}\right)<\frac{\varepsilon_{0}}{k}, y\left(t_{q}^{(k)+}, \phi_{k}\right) \leq \frac{\varepsilon_{0}}{k^{2}}, \\
\frac{\varepsilon_{0}}{k^{2}} \leq y\left(t, \phi_{k}\right) \leq \frac{\varepsilon_{0}}{k} \text { for all } t \in\left(s_{q}^{(k)}, t_{q}^{(k)}\right) .
\end{gathered}
$$

Let $\quad H^{(k)}=\sup \left\{\left|x_{i}\left(t+s, \phi_{k}\right), y\left(t+s, \phi_{k}\right)\right|: t \in R_{+}, s \in R_{-}, i=1,2\right\} \quad$ for $\quad$ each $k=K+1, K+2, \cdots$, we choose a constant $\tau^{(k)}>0$ such that

$$
\int_{-\infty}^{-\tau^{(k)}} k(s) x_{1}\left(t+s, \phi_{k}\right) \mathrm{d} s \leq H^{(k)} \int_{-\infty}^{-\tau^{(k)}} k(s) \mathrm{d} s<\varepsilon_{0},
$$

by (3.20), for each $k=K+1, K+2, \cdots$, there is a $T_{1}^{(k)}>T$ such that

$$
x_{1}\left(t, \phi_{k}\right) \leq x_{1}^{*}(t)+\varepsilon_{0} \text { for all } t \geq T_{1}^{(k)} \text {. }
$$

Clearly, from (3.22), there is an $N_{1}^{(k)}>0$ such that $s_{q}^{(k)}>T_{1}^{(k)}+\tau^{(k)}$ for $q \geq N_{1}^{(k)}$. Hence for any $t \in\left[s_{q}^{k}, t_{q}^{k}\right]$ and $t \neq t_{l}, \quad q \geq N_{1}^{(k)}$. By (3.27) and (3.28), we can obtain 


$$
\begin{aligned}
y^{\prime}\left(t, \phi_{k}\right)= & y\left(t, \phi_{k}\right)\left(a_{3}(t)-b_{3}(t) y\left(t, \phi_{k}\right)-c_{2}(t) \int_{-\infty}^{0} k_{2}(s) x_{1}\left(t+s, \phi_{k}\right) \mathrm{d} s\right) \\
\geq & y\left(t, \phi_{k}\right)\left(a_{3}(t)-b_{3}(t) y\left(t, \phi_{k}\right)-c_{2}(t)\left(\int_{-\infty}^{-\tau^{(k)}} k_{2}(s) x_{1}\left(t+s, \phi_{k}\right) \mathrm{d} s\right.\right. \\
& \left.\left.+\int_{-\tau^{(k)}}^{0} k_{2}(s)\left(x_{1}^{*}(t+s)+\varepsilon_{0}\right) \mathrm{d} s\right)\right) \\
\geq & y\left(t, \phi_{k}\right)\left(a_{3}(t)-b_{3}(t) \varepsilon_{0}-c_{2}(t)\left(H^{(k)} \int_{-\infty}^{-\tau^{(k)}} k_{2}(s) \mathrm{d} s\right.\right. \\
& \left.\left.+\int_{-\tau^{(k)}}^{0} k_{2}(s)\left(x_{1}^{*}(t+s)+\varepsilon_{0}\right) \mathrm{d} s\right)\right) \\
\geq & y\left(t, \phi_{k}\right)\left(a_{3}(t)-b_{3}(t) \varepsilon_{0}-2 c_{2}(t) \varepsilon_{0}-\int_{-\infty}^{0} k_{2}(s) x_{1}^{*}(t+s) \mathrm{d} s\right),
\end{aligned}
$$

choose an integer $l_{q}^{(k)}$ such that $t_{q}^{(k)}=s_{q}^{k}+l_{q}^{k} \bar{\omega}$. Integrating above inequality from $s_{q}^{(k)}$ to $t_{q}^{(k)}$, we obtain

$$
\begin{aligned}
y\left(t_{q}^{(k)+}, \phi_{k}\right) \geq & y\left(s_{q}^{(k)}, \phi_{k}\right) \exp \int_{s_{q}^{(k)}}^{t_{q}^{(k)}}\left(a_{3}(t)-b_{3}(t) \varepsilon_{0}-2 c_{2}(t) \varepsilon_{0}\right. \\
& \left.-\int_{-\tau^{(k)}}^{0} k_{2}(s) x_{1}^{*}(t+s) \mathrm{d} s+\sum_{s_{q}^{(k)} \leq t_{k} \leq t_{q}^{(k)}} \ln g_{k}\right) \\
\geq & y\left(s_{q}^{(k)}, \phi_{k}\right) \exp \int_{s_{q}^{(k)}}^{t_{q}^{(k)}}(-\beta-2 G)
\end{aligned}
$$

Consequently, from (3.25) and (3.26), we have

$$
t_{q}^{(k)}-s_{q}^{(k)} \geq \frac{\ln k}{\beta+2 G}
$$

For any $t \geq s_{q}^{(k)}$ and $q \geq N_{1}^{(k)}$, we have

$$
\int_{-\infty}^{T_{1}^{(k)}} k_{2}(u-t) y\left(u, \phi_{k}\right) \mathrm{d} u \leq H^{(k)} \int_{-\infty}^{T_{1}^{(k)}-t} k_{2}(s) \mathrm{d} s
$$

and

$$
\int_{T_{1}^{(k)}}^{s_{q}^{(k)}} k_{2}(u-t) y\left(u, \phi_{k}\right) \mathrm{d} u \leq H \int_{-\infty}^{s_{q}^{(k)}-t} k_{2}(s) \mathrm{d} s .
$$

For each $k=K+1, K+2, \cdots$, there exist an $N_{2}^{k} \geq N_{1}^{k}$ and a constant $L>0$ such that

$$
H^{(k)} \int_{-\infty}^{T_{1}^{(k)}-s_{q}^{(k)}} k_{2}(s) \mathrm{d} s \leq \frac{1}{2} \varepsilon_{0} \text { for all } q \geq N_{2}^{(k)}
$$

and

$$
H \int_{-\infty}^{-L} k_{2}(s) \mathrm{d} s \leq \frac{1}{2} \varepsilon_{0}
$$

We can choose an integer $r_{q}^{(k)} \geq 0$ such that $t_{q}^{(k)}=s_{q}^{(k)}+L+r_{q}^{(k)} \bar{\omega}+w_{q}^{(k)}$, where $w_{q}^{(k)} \in[0, \bar{\omega})$ is a constant. By (3.30), there exists a large enough $K_{1} \geq K$ such that

$$
r_{q}^{(k)} \varepsilon_{0}-\beta \bar{\omega}-2 G \geq \varepsilon_{0}
$$


For all $k \geq K_{1}, q \geq N_{2}^{(k)}, \quad t \in\left[s_{q}^{(k)}+L, t_{q}^{(k)}\right]$ and $t \neq t_{l}$, by (3.27) and (3.31)-(3.34), we have

$$
\begin{aligned}
& y^{\prime}\left(t, \phi_{k}\right) \\
& \geq y\left(t, \phi_{k}\right)\left(a_{3}(t)-b_{3}(t) y\left(t, \phi_{k}\right)-c_{2}(t)\left(\int_{-\infty}^{T_{1}^{(k)}}+\int_{T_{1}^{k}}^{s_{q}^{(k)}}+\int_{s_{q}^{(k)}}^{t} k_{2}(u-t) x_{1}\left(u, \phi_{k}\right) \mathrm{d} u\right)\right) \\
& \geq y\left(t, \phi_{k}\right)\left(a_{3}(t)-b_{3}(t) \frac{\varepsilon_{0}}{k}-c_{2}(t)\left(H^{(k)} \int_{-\infty}^{T_{1}^{(k)}-t} k_{2}(s) \mathrm{d} s\right.\right. \\
& \left.\left.+H \int_{-\infty}^{s_{q}^{(k)}-t} k_{2}(s) \mathrm{d} s+\int_{s_{q}^{(k)}}^{t} k_{2}(u-t)\left(x_{1}^{*}(u)+\varepsilon_{0}\right) \mathrm{d} u\right)\right) \\
& \quad \geq y\left(t, \phi_{k}\right)\left(a_{3}(t)-b_{3}(t) \varepsilon_{0}-c_{2}(t)\left(\frac{\varepsilon_{0}}{2}+\frac{\varepsilon_{0}}{2}+\varepsilon_{0}+\int_{-t}^{0} k(s) x_{1}^{*}(t+s) \mathrm{d} s\right)\right) \\
& \quad=y\left(t, \phi_{k}\right)\left(a_{3}(t)-b_{3}(t) \varepsilon_{0}-2 c_{2}(t) \varepsilon_{0}-c_{2}(t) \int_{-t}^{0} k_{2}(s) x_{1}^{*}(t+s) \mathrm{d} s\right) .
\end{aligned}
$$

Integrating above system from $s_{q}^{(k)}+L$ to $t_{q}^{(k)}$, we derive

$$
\begin{aligned}
\frac{\varepsilon_{0}}{k^{2} \geq} & y\left(t_{q}^{(k)+}, \phi_{k}\right) \\
\geq & y\left(s_{q}^{(k)}+L, \phi_{k}\right) \exp \left(\int_{s_{q}^{(k)}+L}^{s_{q}^{(k)}+L+\bar{\omega}}+\cdots+\int_{s_{q}^{(k)}+L+\left(r_{q}^{(k)}-1\right) \bar{\omega}}^{s_{(k)}^{(k)}+L+\int_{s_{q}}^{(k)} \overline{(k)}+L+r_{q}^{(k)} \omega} t_{3}^{(k)}\left(a_{3}(t)-b_{3}(t) \varepsilon_{0}\right.\right. \\
& \left.-2 c_{2}(t) \varepsilon_{0}-c(t) \int_{-t}^{0} k_{2}(s)\left(x_{1}^{*}(t+s)\right) \mathrm{d} s\right) \mathrm{d} s \\
& \left.+\left(\sum_{s_{q}^{(k)}+L \leq t \leq s_{q}^{(k)}+L+\bar{\omega}}+\cdots+\sum_{s_{q}^{(k)}+L+\left(r_{q}^{(k)}-1\right) \bar{\omega} \leq t \leq s_{q}^{(k)}+L+r_{q}^{(k)} \bar{\omega}}+\sum_{s_{q}^{(k)}+L+r_{q}^{(k)} \bar{\omega} \leq t \leq t_{q}^{(k)}} \ln g_{k}\right)\right) \\
\geq & \frac{\varepsilon_{0}}{k^{2}} \exp \left(r_{q}^{(k)} \varepsilon_{0}-\beta \bar{\omega}-2 G\right) \geq \frac{\varepsilon_{0}}{k^{2}} \exp \left(\varepsilon_{0}\right)>\frac{\varepsilon_{0}}{k^{2}},
\end{aligned}
$$

which is a contradiction. This contradiction shows that there exists a constant $m_{2}>0$ such that $\liminf _{t \rightarrow \infty} y(t) \geq m_{2}$. Hence, choose a constant $m=\min \left\{m_{1}, m_{2}\right\}$, then we finally have

$$
\liminf _{t \rightarrow \infty} x_{i}(t) \geq m(i=1,2), \liminf _{t \rightarrow \infty} y(t) \geq m
$$

Therefore Theorem 3.2 holds. This completes the proof.

\section{Extinction}

In this section, we investigate the extinction of system (1.1). We note that, under conditions of Theorem 3.2, system (1.1) is always permanent.

Theorem 4.1. Assume that there is a constant $\eta \geq 0$ such that

$$
\begin{gathered}
\limsup _{t \rightarrow \infty}\left(\int_{t}^{t+\eta} \gamma(s) \mathrm{d} s+\sum_{t \leq t_{k}<t+\eta} \ln h_{k}\right) \leq 0 \\
\limsup _{t \rightarrow \infty}\left(\int_{t}^{t+\eta}\left(a_{3}(s)-m c_{2}(s)\right) \mathrm{d} s+\sum_{t \leq t_{k}<t+\eta} \ln g_{k}\right) \leq 0
\end{gathered}
$$

and function 


$$
\begin{aligned}
& h(t, \mu)=\sum_{t \leq t_{k}<t+\mu} \ln h_{k} \\
& g(t, \mu)=\sum_{t \leq t_{k}<t+\mu} \ln g_{k}
\end{aligned}
$$

are bounded function on $t \in R_{+}$and $v \in[0, \eta)$, where $\gamma(t)=a_{1}(t)+a_{2}(t)+D(t)$, then we can obtain

$$
\lim _{t \rightarrow \infty} x_{i}(t)=0, \quad \lim _{t \rightarrow \infty} y(t)=0, \quad(i=1,2)
$$

for any positive solution $\left(x_{1}(t), x_{2}(t), y(t)\right)$ of system (1.1).

Proof. Firstly, we prove the extinction of species $x$. Define $V(t)=x_{1}(t)+x_{2}(t)$. When $t \neq t_{k}$, calculating the right-upper derivative of $V(t)$, we have

$$
\begin{aligned}
& D^{+} V(t)= x_{1}^{\prime}(t)+x_{2}^{\prime}(t) \\
&= x_{1}(t)\left(a_{1}(t)-b_{1}(t) x_{1}(t)-c_{1}(t) \int_{-\infty}^{0} k_{1}(s) y(t+s) \mathrm{d} s\right) \\
&+D_{12}(t)\left(x_{2}(t)-x_{1}(t)\right)+x_{2}(t)\left(a_{2}(t)-b_{2}(t) x_{2}(t)\right) \\
&+D_{21}(t)\left(x_{1}(t)-x_{2}(t)\right) \\
& \leq x_{1}(t)\left(a_{1}(t)-b_{1}(t) x_{1}(t)-D_{12}(t)+D_{21}(t)\right) \\
&+ x_{2}(t)\left(a_{2}(t)-b_{2}(t) x_{2}(t)-D_{21}(t)+D_{12}(t)\right) \\
& \leq\left(x_{1}(t)+x_{2}(t)\right)\left(a_{1}(t)-b_{1}(t) x_{1}(t)+D(t)\right) \\
&+\left(x_{1}(t)+x_{2}(t)\right)\left(a_{2}(t)-b_{2}(t) x_{2}(t)+D(t)\right) \\
& \leq\left(x_{1}(t)+x_{2}(t)\right)\left(a_{1}(t)+a_{2}(t)+2 D(t)-2 \bar{b}(t)\left(x_{1}(t)+x_{2}(t)\right)\right) \\
&=V(t)(\gamma(t)-2 \bar{b}(t) V(t)),
\end{aligned}
$$

when $t=t_{k}$, we get

$$
V\left(t_{k}^{+}\right)=x_{1}\left(t_{k}^{+}\right)+x_{2}\left(t_{k}^{+}\right)=h_{1 k} x_{1}\left(t_{k}\right)+h_{2 k} x_{2}\left(t_{k}\right) \leq \max \left\{h_{1 k}, h_{2 k}\right\} V\left(t_{k}\right)=h_{k} V\left(t_{k}\right)
$$

By the comparison theory of impulsive differential equations, we have $V(t) \leq v(t)$ for all $t \geq 0$, where $v(t)$ is a solution of the auxiliary equation

$$
\left\{\begin{array}{l}
v^{\prime}(t)=v(t)(\gamma(t)-2 \bar{b}(t) v(t)), t \neq t_{k} \\
v\left(t_{k}^{+}\right)=h_{k} v\left(t_{k}\right), k=1,2, \cdots
\end{array}\right.
$$

with the initial condition $v(0)=V(0)$. Since system (4.6) satisfies all conditions of Lemma 2.1 from conditions (4.1) and (4.3), we obtain $\lim _{t \rightarrow \infty} v(t)=0$ for any positive solution of system (4.6). Then we get

$$
\lim _{t \rightarrow \infty} x_{i}(t) \leq \lim _{t \rightarrow \infty} V(t) \leq \lim _{t \rightarrow \infty} v(t)=0,(i=1,2) .
$$

In the following, we prove the extinction of species $y$. From third equation and sixth equation of system (1.1), we have

$$
\left\{\begin{array}{l}
y^{\prime}(t)=y(t)\left(a_{3}(t)-b_{3}(t) y(t)-c_{2}(t) \int_{-\infty}^{0} k_{2}(s) x_{1}(t-s) \mathrm{d} s\right), t \neq t_{k} \\
y\left(t_{k}^{+}\right)=g_{k} y\left(t_{k}\right), k=1,2, \cdots
\end{array}\right.
$$

By Theorem 3.2, there exist $m>0$ and $T_{0}>0$ such that $x_{1}(t)>m$ for all 
$t \geq T_{0}$. Obviously, when $t \neq t_{k}$, for any $t \geq T_{0}$, we obtain

$$
\begin{aligned}
y^{\prime}(t) & =y(t)\left(a_{3}(t)-b_{3}(t) y(t)-c_{2}(t) \int_{-\infty}^{0} k_{2}(s) x_{1}(t-s) \mathrm{d} s\right) \\
& \leq y(t)\left(a_{3}(t)-b_{3}(t) y(t)-m c_{2}(t)\right)
\end{aligned}
$$

Then we consider the following auxiliary system

$$
\left\{\begin{array}{l}
w^{\prime}(t)=w(t)\left(a_{3}(t)-b_{3}(t) w(t)-m c_{2}(t)\right), t \neq t_{k} \\
w\left(t_{k}^{+}\right)=g_{k} w\left(t_{k}\right), k=1,2, \cdots
\end{array}\right.
$$

with the initial condition $w\left(T_{0}\right)=y\left(T_{0}\right)$. Since all condition of Lemma 2.1 holds from condition (4.2) and (4.4), we can obtain $\lim _{t \rightarrow \infty} w(t)=0$ for any positive solution of system (4.9). Clearly, we have

$$
\lim _{t \rightarrow \infty} y(t) \leq \lim _{t \rightarrow \infty} w(t)=0 .
$$

From (4.7) and (4.10), we finally obtain (4.5) holds. This completes the proof of Theorem 4.1 .

\section{Globally Attractive}

In this section, by constructing appropriate Lyapunov function, we establish the sufficient conditions on the globally attractive of system (1.1).

Theorem 5.1. Assume that all conditions of Theorem 3.2 hold, further, there exists a constant $\lambda>0$ such that

$$
\liminf _{t \rightarrow \infty}\left(\int_{t}^{t+\lambda} \psi(s) \mathrm{d} s\right)>0,
$$

where

$$
\begin{aligned}
\psi(t)=\min _{t \in R^{+}} & \left\{b_{1}(t)-\frac{D_{21}(t)}{m_{1}}-\int_{0}^{+\infty} k_{2}(s) c_{2}(t-s) \mathrm{d} s,\right. \\
& \left.b_{2}(t)-\frac{D_{12}(t)}{m_{1}}, b_{3}(t)-\int_{0}^{+\infty} k_{1}(s) c_{1}(t-s) \mathrm{d} s\right\}
\end{aligned}
$$

and $\psi(t) \geq 0$. Then system (1.1) is globally attractive, that is, for any two positive solutions $\left(x_{1}(t), x_{2}(t), y(t)\right)$ and $\left(\tilde{x}_{1}(t), \tilde{x}_{2}(t), \tilde{y}(t)\right)$ of system (1.1), the following limit hold.

$$
\lim _{t \rightarrow \infty}\left|x_{1}(t)-\tilde{x}_{1}(t)\right|=0, \lim _{t \rightarrow \infty}\left|x_{2}(t)-\tilde{x}_{2}(t)\right|=0, \lim _{t \rightarrow \infty}|y(t)-\tilde{y}(t)|=0 .
$$

Proof. For any two positive solutions $\left(x_{1}(t), x_{2}(t), y(t)\right),\left(\tilde{x}_{1}(t), \tilde{x}_{2}(t), \tilde{y}(t)\right)$, by Theorem 3.2, we obtain that there exist constants $m_{1}, M_{1}$ such that

$$
m_{1} \leq x_{i}(t), \tilde{x}_{i}(t), y(t), \tilde{y}(t) \leq M_{1}, i=1,2 .
$$

Then we have for any $t \in R_{+}$and $t \neq t_{k}$,

$$
\begin{gathered}
\frac{1}{m_{1}}\left|x_{i}(t)-\tilde{x}_{i}(t)\right| \leq\left|\ln x_{i}(t)-\ln \tilde{x}_{i}(t)\right| \leq \frac{1}{M_{1}}\left|x_{i}(t)-\tilde{x}_{i}(t)\right|(i=1,2) \\
\frac{1}{m_{1}}|y(t)-\tilde{y}(t)| \leq|\ln y(t)-\ln \tilde{y}(t)| \leq \frac{1}{M_{1}}|y(t)-\tilde{y}(t)|
\end{gathered}
$$

Define a Lyapunov function 


$$
V_{1}(t)=\left|\ln x_{1}(t)-\ln \tilde{x}_{1}(t)\right|+\left|\ln x_{2}(t)-\ln \tilde{x}_{2}(t)\right|+|\ln y(t)-\ln \tilde{y}(t)|
$$

for any impulsive time $t_{k}$, we have

$$
V_{1}\left(t_{k}\right)=\sum_{i=1}^{2}\left|\ln h_{i k} x_{i}\left(t_{k}\right)-\ln h_{i k} \tilde{x}_{i}\left(t_{k}\right)\right|+\left|\ln g_{k} y\left(t_{k}\right)-\ln g_{k} \tilde{y}\left(t_{k}\right)\right|=V_{1}\left(t_{k}^{+}\right),
$$

$V_{1}(t)$ is continuous for all $t \in R_{+}$. For any $t \in R_{+}$and $t \neq t_{k}$, calculating the derivative of $V_{1}(t)$, then we get

$$
\begin{aligned}
& D^{+} V_{1}(t)=\operatorname{sgn}\left(x_{1}(t)-\tilde{x}_{1}(t)\right)\left(\frac{x_{1}^{\prime}(t)}{x_{1}(t)}-\frac{\tilde{x}_{1}^{\prime}(t)}{\tilde{x}_{1}(t)}\right) \\
& +\operatorname{sgn}\left(x_{2}(t)-\tilde{x}_{2}(t)\right)\left(\frac{x_{2}^{\prime}(t)}{x_{2}(t)}-\frac{\tilde{x}_{2}^{\prime}(t)}{\tilde{x}_{2}(t)}\right)+\operatorname{sgn}(y(t)-\tilde{y}(t))\left(\frac{y^{\prime}(t)}{y(t)}-\frac{\tilde{y}^{\prime}(t)}{\tilde{y}(t)}\right) \\
& \leq \operatorname{sgn}\left(x_{1}(t)-\tilde{x}_{1}(t)\right)\left[-b_{1}(t)\left(x_{1}(t)-\tilde{x}_{1}(t)\right)\right. \\
& \left.-c_{1}(t) \int_{0}^{+\infty} k_{1}(s)(y(t-s)-\tilde{y}(t-s)) \mathrm{d} s+D_{12}(t)\left(\frac{x_{2}(t)}{x_{1}(t)}-\frac{\tilde{x}_{2}(t)}{\tilde{x}_{1}(t)}\right)\right] \\
& +\operatorname{sgn}\left(x_{2}(t)-\tilde{x}_{2}(t)\right)\left[\left(-b_{2}(t)\left(x_{1}(t)-\tilde{x}_{1}(t)\right)\right)+D_{21}(t)\left(\frac{x_{1}(t)}{x_{2}(t)}-\frac{\tilde{x}_{1}(t)}{\tilde{x}_{2}(t)}\right)\right] \\
& +\operatorname{sgn}(y(t)-\tilde{y}(t))\left[-b_{3}(t)(y(t)-\tilde{y}(t))\right. \\
& \left.-c_{2}(t) \int_{0}^{+\infty} k_{2}(s)\left(x_{1}(t-s)-\tilde{x}_{1}(t-s)\right) \mathrm{d} s\right]
\end{aligned}
$$

Let

$$
\begin{aligned}
& \bar{D}_{12}(t)=\operatorname{sgn}\left(x_{1}(t)-\tilde{x}_{1}(t)\right) D_{12}(t)\left(\frac{x_{2}(t)}{x_{1}(t)}-\frac{\tilde{x}_{2}(t)}{\tilde{x}_{1}(t)}\right), \\
& \bar{D}_{21}(t)=\operatorname{sgn}\left(x_{2}(t)-\tilde{x}_{2}(t)\right) D_{21}(t)\left(\frac{x_{1}(t)}{x_{2}(t)}-\frac{\tilde{x}_{1}(t)}{\tilde{x}_{2}(t)}\right) .
\end{aligned}
$$

for $\bar{D}_{12}(t), \bar{D}_{21}(t)$, we consider the following cases:

1) If $x_{i}(t)>\tilde{x}_{i}(t)(i=1,2)$ for all $t \geq 0$, then

$\bar{D}_{12}(t) \leq D_{12}(t)\left(\frac{x_{2}(t)}{x_{1}(t)}-\frac{\tilde{x}_{2}(t)}{\tilde{x}_{1}(t)}\right) \leq \frac{D_{12}(t)}{x_{1}(t)}\left(x_{2}(t)-\tilde{x}_{2}(t)\right) \leq \frac{D_{12}(t)}{m_{1}}\left|x_{2}(t)-\tilde{x}_{2}(t)\right|$,

$\bar{D}_{21}(t) \leq D_{21}(t)\left(\frac{x_{1}(t)}{x_{2}(t)}-\frac{\tilde{x}_{1}(t)}{\tilde{x}_{2}(t)}\right) \leq \frac{D_{21}(t)}{x_{2}(t)}\left(x_{1}(t)-\tilde{x}_{1}(t)\right) \leq \frac{D_{21}(t)}{m_{1}}\left|x_{1}(t)-\tilde{x}_{1}(t)\right|$.

2) If $x_{i}(t)<\tilde{x}_{i}(t)(i=1,2)$ for all $t \geq 0$, then

$\bar{D}_{12}(t) \leq D_{12}(t)\left(\frac{\tilde{x}_{2}(t)}{\tilde{x}_{1}(t)}-\frac{x_{2}(t)}{x_{1}(t)}\right) \leq \frac{D_{12}(t)}{\tilde{x}_{1}(t)}\left(\tilde{x}_{2}(t)-x_{2}(t)\right) \leq \frac{D_{12}(t)}{m_{1}}\left|x_{2}(t)-\tilde{x}_{2}(t)\right|$,

$\bar{D}_{21}(t) \leq D_{21}(t)\left(\frac{\tilde{x}_{1}(t)}{\tilde{x}_{2}(t)}-\frac{x_{1}(t)}{x_{2}(t)}\right) \leq \frac{D_{21}(t)}{\tilde{x}_{2}(t)}\left(\tilde{x}_{1}(t)-x_{1}(t)\right) \leq \frac{D_{21}(t)}{m_{1}}\left|x_{1}(t)-\tilde{x}_{1}(t)\right|$.

3) If $x_{i}(t)=\tilde{x}_{i}(t)(i=1,2)$ for all $t \in R_{+}$, similar to the arguments above, we 
can get the same conclusion as (1) and (2). From (1), (2) and (3), we have

$$
\begin{aligned}
& \bar{D}_{12}(t) \leq \frac{D_{12}(t)}{m_{1}}\left|x_{2}(t)-\tilde{x}_{2}(t)\right|, \\
& \bar{D}_{21}(t) \leq \frac{D_{21}(t)}{m_{1}}\left|x_{1}(t)-\tilde{x}_{1}(t)\right| .
\end{aligned}
$$

Due to (5.6) and (5.7), we can obtain

$$
\begin{aligned}
D^{+} V_{1}(t) \leq & -b_{1}(t)\left|x_{1}(t)-\tilde{x}_{1}(t)\right|+c_{1}(t) \int_{0}^{+\infty} k_{1}(s)|y(t-s)-\tilde{y}(t-s)| \mathrm{d} s \\
& +\frac{D_{12}(t)}{m_{1}}\left|x_{2}(t)-\tilde{x}_{2}(t)\right|+-b_{2}(t)\left|x_{1}(t)-\tilde{x}_{1}(t)\right| \\
& +\frac{D_{21}(t)}{m_{1}}\left|x_{1}(t)-\tilde{x}_{1}(t)\right|+-b_{3}(t)|y(t)-\tilde{y}(t)| \\
& +c_{2}(t) \int_{0}^{+\infty} k_{2}(s)\left|x_{1}(t-s)-\tilde{x}_{1}(t-s)\right| \mathrm{d} s .
\end{aligned}
$$

Moreover, we define

$$
\begin{aligned}
& V_{2}(t)=\int_{0}^{+\infty} k_{1}(s) \int_{t-s}^{t} c_{1}(u-s)|y(u)-\tilde{y}(u)| \mathrm{d} u \mathrm{~d} s, \\
& V_{3}(t)=\int_{0}^{+\infty} k_{2}(s) \int_{t-s}^{t} c_{2}(u-s)\left|x_{1}(u)-\tilde{x}_{1}(u)\right| \mathrm{d} u \mathrm{~d} s .
\end{aligned}
$$

Obviously, $V_{2}(t)$ and $V_{3}(t)$ are continuous for all $t \geq 0$ and $t \neq t_{k}$. Calculating the upper right derivative, we derive that

$$
\begin{aligned}
D^{+} V_{2}(t)= & \int_{0}^{+\infty} k_{1}(s) c_{1}(t-s)|y(t)-\tilde{y}(t)| \mathrm{d} s \\
& -\int_{0}^{+\infty} k_{1}(s) c_{1}(t)|y(t-s)-\tilde{y}(t-s)| \mathrm{d} s, \\
D^{+} V_{3}(t)= & \int_{0}^{+\infty} k_{2}(s) c_{2}(t-s)\left|x_{1}(t)-\tilde{x}_{1}(t)\right| \mathrm{d} s \\
& -\int_{0}^{+\infty} k_{1}(s) c_{2}(t)\left|x_{1}(t-s)-\tilde{x}_{1}(t-s)\right| \mathrm{d} s .
\end{aligned}
$$

Define $V(t)=V_{1}(t)+V_{2}(t)+V_{3}(t)$, then we can follows from (5.8) and (5.9) that

$$
\begin{aligned}
D^{+} V(t)= & D^{+} V_{1}(t)+D^{+} V_{2}(t)+D^{+} V_{3}(t) \\
\leq & -b_{1}(t)\left|x_{1}(t)-\tilde{x}_{1}(t)\right|+\int_{0}^{+\infty} k_{1}(s) c_{1}(t-s)|y(t)-\tilde{y}(t)| \mathrm{d} s \\
& +\frac{D_{12}(t)}{m_{1}}\left|x_{2}(t)-\tilde{x}_{2}(t)\right|-b_{2}(t)\left|x_{2}(t)-\tilde{x}_{2}(t)\right| \\
& \quad+\frac{D_{21}(t)}{m_{1}}\left|x_{1}(t)-\tilde{x}_{1}(t)\right|-b_{3}(t)|y(t)-\tilde{y}(t)| \\
& \quad+\int_{0}^{+\infty} k_{2}(s) c_{2}(t-s)\left|x_{1}(t)-\tilde{x}_{1}(t)\right| \mathrm{d} s \\
\leq- & \left(b_{1}(t)-\frac{D_{21}(t)}{m_{1}}-\int_{0}^{+\infty} k_{2}(s) c_{2}(t-s) \mathrm{d} s\right)\left|x_{1}(t)-\tilde{x}_{1}(t)\right| \\
& -\left(b_{2}(t)-\frac{D_{12}(t)}{m_{1}}\right)\left|x_{2}(t)-\tilde{x}_{2}(t)\right| \\
& -\left(b_{3}(t)-\int_{0}^{+\infty} k_{1}(s) c_{1}(t-s) \mathrm{d} s\right)|y(t)-\tilde{y}(t)| \\
\leq & -\psi(t) m_{1} V(t) .
\end{aligned}
$$

Integrating above inequality, we further have 
$V(t) \leq V(0) \exp \left(-m_{1} \int_{0}^{t} \psi(s) \mathrm{d} s\right)$ for all $t \in R_{+}$. Then from (5.1) we have $\int_{0}^{t} \psi(s) \mathrm{d} s \rightarrow \infty$ as $t \rightarrow \infty$. Thus, we have $V(t) \rightarrow 0$ as $t \rightarrow \infty$. Finally, from (5.4) and (5.5) we know (5.2) holds. This completes the proof of Theorem 5.1.

\section{Conclusions}

In this paper, we investigated an impulsive competitive system with infinite delay and diffusion, in which $x_{1}$ can disperse between patch 1 and patch 2 , but competitor $y$ is confined to patch 1 . We also gave some sufficient conditions on permanence, extinction and global attractivity of system (1.1). From Theorem 3.1-Theorem 5.1, we can see that the impulse and dispersal have an influence on permanence, extinction and global attractivity. Moreover, we note that the infinite delay is harmless for the extinction, but it affects the permanence and global attractivity of system (1.1).

Further, we can observe that impulsive perturbations play an important role in the permanence and extinction from Theorem 3.1-Theorem 4.1. In ecological environment, many natural and man-made factors which can be described impulse in mathematical always lead to rapid decrease or increase of the population number. So we consider the following two cases.

Theorem 3.1 shows that if the density-coefficients $b_{i}(t)(i=1,2)$ are greater than zero and the impulsive coefficients $h_{i k}(i=1,2)$ are bounded, the species $x$ and $y$ are always ultimately bounded. In following discussion, we also assume that satisfies this condition.

Discuss 1 On condition that the impulses lead to decrease of the number of species (such as fire, drought, hunting, harvesting, flooding deforestation), then the impulsive coefficients satisfy $0<h_{i k} \leq 1$ and $0<g_{k} \leq 1$ for all $(i=1,2, k=1,2, \cdots)$.

1) Theorem 3.2 shows that if the impulsive perturbations $h_{1 k}, h_{2 k}$ are relatively small compared to the intrinsic growth rate of $x$, the species $x$ can keep permanence; if the impulsive perturbations $g_{k}$ are relatively small, in addition, the delay, competition coefficients of $y$ and dispersal coefficients of $x$ relatively small make the intrinsic growth rate of $y$ to increase, then the species $y$ keeps permanence.

2) Theorem 4.1 shows that if the impulsive perturbations $h_{1 k}, h_{2 k}$ are relatively large compared to the intrinsic growth rate and dispersal coefficient of $x$, then the species $x$ tends to extinction; if the impulsive perturbations $g_{k}$ are relatively large and the intrinsic growth rate of $y$ is relatively small, the species $y$ tends to extinction.

Discuss 2 On condition that impulses lead to increase of the number of species (such as feed, replenishment, input or other protective measures from human), that is the impulsive coefficients satisfy $h_{1 k}, h_{2 k}, g_{k} \geq 1$ for all $k=1,2, \cdots$.

1) Theorem 3.2 shows that the species $x$ always keep permanence; if the delay, competition coefficients of $y$ and dispersal coefficients of $x$ are relatively small making the intrinsic growth rate of $y$ to increase; regardless of impulsive influence which is large or small, the species $y$ keeps permanence. 
2) Theorem 4.1 shows that the species $x$ never tends to extinction; if the impulsive perturbations $g_{k}$ and the intrinsic growth rate are relatively small; besides, the competition coefficient is relatively large, then the species $y$ tends to extinction.

\section{Acknowledgements}

This paper is supported by Natural Science Foundation of Guangxi (2016GXNSFAA380194).

\section{References}

[1] Bainov, D.D. and Simeonov, P.S. (1993) Impulsive Differential Equations: Periodic Solutions and Applications. Longman, Harlow.

[2] Akman, O., Comar, T. and Henderson, M. (2018) An Analysis of an Impulsive Stage Structured Integrated Pest Management Model with Refuge Effect. Chaos, Solitons \& Fractals, 111, 44-54. https://doi.org/10.1016/j.chaos.2018.03.039

[3] He, M., Chen, F. and Li, Z. (2016) Permanence and Global Attractivity of an Impulsive Delay. Applied Mathematics Letters, 62, 92-100.

https://doi.org/10.1016/j.aml.2016.07.009

[4] Shao, Y.F., Dai, B.X. and Luo, Z.G. (2010) The Dynamics of an Impulsive One-Prey Multi-Predators System with Delay and Holling-Type II Functional Response. Applied Mathematics and Computation, 217, 2414-2424. https://doi.org/10.1016/j.amc.2010.07.042

[5] He, M.X., Chen, F.D. and Li, Z. (2016) Permanence and Global Attractivity of an Impulsive Delay Logistic Model. Applied Mathematics Letters, 62, 92-100. https://doi.org/10.1016/j.aml.2016.07.009

[6] Tan, R.H., Liu, W.F., Wang, Q.L. and Liu, Z.J. (2014) Uniformly Asymptotic Stability of Almost Periodic Solutions for a Competitive System with Impulsive Perturbations. Advances in Difference Equations, 2014, 2.

https://doi.org/10.1186/1687-1847-2014-2

[7] Ut, V. and Le, E.P. (2013) A Model for the Problem of the Cooperation/Competition between Infinite Continuous Species. Ricerche Di Matematica, 62, 139-153. https://doi.org/10.1007/s11587-013-0148-6

[8] Hu, H.X., Wang, K. and Wu, D. (2011) Permanence and Global Stability for Nonautonomous N-Species Lotka-Volterra Competitive System with Impulses and Infinite Delays. Journal of Mathematical Analysis \& Applications, 377, 145-160. https://doi.org/10.1016/j.jmaa.2010.10.031

[9] Shao, Y.F. (2012) Globally Asymptotical Stability and Periodicity for a Nonautonomous Two-Species System with Diffusion and Impulses. Applied Mathematical Modelling, 36, 288-300. https://doi.org/10.1016/j.apm.2011.05.044

[10] Wang, W.B., Shen, J.H. and Luo, Z.G. (2009) Partial Survival and Extinction in Two Competing Species with Impulses. Nonlinear Analysis Real World Applications, 10, 1243-1254. https://doi.org/10.1016/j.nonrwa.2007.11.012

[11] Liu, Z.J. and Zhong, S.M. (2012) An Impulsive Periodic Predator-Prey System with Holling Type III Functional Response and Diffusion. Applies Mathematical Modelling, 36, 5967-5990. https://doi.org/10.1016/j.apm.2012.01.032

[12] Liu, Z.J., Zhong, S.M., Teng, Z.D. and Zhang, L. (2013) Permanence and Global Attractivity of Impulsive Ratio-Dependent Predator-Prey System in a Patchy Envi- 
ronment. Applied Mathematics and Computation, 219, 9791-9804. https://doi.org/10.1016/j.amc.2013.03.108

[13] Liu, Z.J. and Zhong, S.M. (2010) Permanence and Extinction Analysis for a Delayed Periodic Predator-Prey System with Holling Type II Response Function and Diffusion. Applied Mathematics and Computation, 216, 3002-3015. https://doi.org/10.1016/j.amc.2010.04.012

[14] Cai, L.M., Li, X.Z., Yu, J.Y. and Zhu, G.T. (2009) Dynamic of a Nonautonomous Predator-Prey Dispersion-Delay System with Bedding-DeAngelis Functional Response. Chaos, Solitons and Fractals, 40, 2064-2075. https://doi.org/10.1016/j.chaos.2007.09.082

[15] Zhang, L. and Teng, Z.D. (2008) Permanence for a Delayed Periodic Predator-Prey Model with Prey Dispersal in Multi-Patches and Predator Density-Independent. Journal of Mathematical Analysis \& Applications, 338, 175-193.

https://doi.org/10.1016/j.jmaa.2007.05.016

[16] Yang, X.X., Wang, W.B. and Shen, J.H. (2011) Permanence of a Logistic Type Impulsive Equation with Infinite Delay. Applied Mathematics Letters, 24, 420-427. https://doi.org/10.1016/j.aml.2010.10.026

[17] Teng, Z.D. and Chen, L.S. (2003) Permanence and Extinction of Periodic Predator-Prey Systems in a Patchy Environment with Delay. Nonlinear Analysis Real World Applications, 4, 335-364. https://doi.org/10.1016/S1468-1218(02)00026-3

[18] Hou, J., Teng, Z.D. and Gao, S.J. (2010) Permanence and Global Stability for Nonautonomous N-Species Lotka-Volterra Competitive System with Impulses. Nonlinear Analysis Real World Applications, 11, 1882-1896.

https://doi.org/10.1016/j.nonrwa.2009.04.012 